\title{
Berberine reverts hepatic mitochondrial dysfunction in high-fat fed rats: A possible role for SirT3 activation
}

\author{
João Soeiro Teodoro ${ }^{\mathrm{a}, \mathrm{b}}$, Filipe Valente Duarte ${ }^{\mathrm{a}, \mathrm{b}}$, Ana Patrícia Gomes ${ }^{\mathrm{a}, \mathrm{b}}$, Ana Teresa Varela ${ }^{\mathrm{a}, \mathrm{b}}$, \\ Francisco Manuel Peixoto ${ }^{c}$, Anabela Pinto Rolo ${ }^{\mathrm{a}, \mathrm{d}}$, Carlos Marques Palmeira ${ }^{\mathrm{a}, \mathrm{b}, *}$ \\ a Center for Neuroscience and Cell Biology, Faculty of Science and Technology, University of Coimbra, 3004-517 Coimbra, Portugal \\ b Department of Life Sciences, Faculty of Science and Technology, University of Coimbra, 3004-517 Coimbra, Portugal \\ c Center for the Research and Technology of Agro-Environmental and Biological Sciences, University of Trás-os-Montes and Alto Douro, 5001-801 Vila Real, Portugal \\ d Department of Biology, University of Aveiro, 3810-193 Aveiro, Portugal
}

\section{A R T I C L E I N F O}

\section{Article history:}

Received 24 May 2013

Received in revised form 6 August 2013

Accepted 6 September 2013

Available online 14 September 2013

\section{Keywords:}

Berberine

Liver bioenergetics

Mitochondrial function

Obesity

SirT3

\begin{abstract}
A B S T R A C T
Berberine is an isoquinoline alkaloid with anti-diabetic properties. Despite the central role of liver and thus hepatic mitochondria in whole-body metabolism, berberine effects on hepatic mitochondrial function in an obesity model are still unknown. Here, we demonstrate that berberine treatment recovers mitochondrial efficiency when altered by a high-fat feeding. Mitochondria isolated from the liver of high-fat fed rats exhibited decreased capacity to accumulate calcium and impaired oxidative phosphorylation (OXPHOS) capacity, as shown by impaired mitochondrial membrane potential, oxygen consumption and cellular ATP levels. Interestingly, the recovery of mitochondrial function by berberine was associated with an increased activity of the mitochondrial sirtuin 3 (SirT3). In conclusion, berberine potent protective effects against metabolic syndrome may rely on increasing mitochondrial SirT3 activity, normalizing mitochondrial function and preventing a state of energetic deficit caused by impaired OXPHOS.
\end{abstract}

(c) 2013 Elsevier B.V. and Mitochondria Research Society. All rights reserved.

\section{Introduction}

Metabolic syndrome, a pre-diabetic condition, is considered a global epidemic due to the elevated incidence of obesity promoted by the western lifestyle and high fat dietary intake (Haslam and James, 2005). This pathological state is a multifactorial condition with elevated morbidity due to the combination of various characteristics, such as abdominal obesity, hyperglycemia, dyslipidemia, insulin resistance, elevated risk of cardiac complications and even cancer (Haslam and James, 2005). This can lead to anomalies in glucose and lipid metabolism throughout the organism, with particular relevance in the liver, muscle and adipocytes (Carley and Severson, 2005). Since the liver occupies a central role in whole-body metabolic regulation, the imbalance of hepatic function by increased availability of nutrients is known to lead to several complications. Intrahepatocytical lipid accumulation has been linked to hepatic and peripheral insulin resistance in type II diabetes (T2D) patients, impaired pancreatic $\beta$-cell function and hepatic

Abbreviations: ACC, acetyl-CoA carboxylase; AMPK, AMP-activated protein kinase; BBR, berberine-supplemented HFD group; Ctl, control low fat diet-fed group; FAME, fatty acid methyl ester; FCCP, carbonylcyanide-P-trifluoromethoxyphenylhydrazon; FFA, free fatty acids; HFD, high fat diet-fed group; NAFLD, non-alcoholic fatty liver disease; OXPHOS, oxidative phosphorylation; RCR, Respiratory Control Ratio; ROS, reactive oxygen species; SirT3, sirtuin 3; T2D, type II diabetes; TPP +, tetraphenylphosphonium.

* Corresponding author at: Department of Life Sciences, Faculty of Science and Technology, University of Coimbra, 3004-517 Coimbra, Portugal. Fax: +351239855 789.

E-mail address: palmeira@ci.uc.pt (C.M. Palmeira). steatosis (Shah et al., 2003). Steatosis is the basis for the induction of liver cirrhosis and inflammatory steatohepatitis (Clark and Diehl, 2003) and can occur in parallel to obesity and/or T2D (Haque and Sanyal, 2002). The hepatic increase in free fatty acids (FFA) leads to mitochondrial dysfunction, ATP depletion and also increased generation of reactive oxygen species (ROS) (Pessayre, 2007).

In the eukaryotic cell, mitochondria are the main ATP generation site. As such, it is of no surprise that altered mitochondrial metabolism is a common hallmark of several pathologies. The excessive FFA accumulation in hepatocytes causes the impairment of mitochondrial bioenergetics that parallels the metabolic evolution of fatty liver disease (Teodoro et al., 2006, 2008). Due to mitochondrial oxidative phosphorylation activity, mitochondria are the prime site of intracellular ROS generation. As such, an imbalance of the cell's redox environment will most likely originate in and affect the cell's mitochondria, despite its natural antioxidant defenses. In fact, it has been reported that, in T2D patients, there is a higher number of damaged mitochondria with reduced mitochondrial volume (Kelley et al., 2002). Concomitantly, this leads to a vicious cycle due to the fact that excessive ROS generation leads to membrane lipid and respiratory chain protein alterations, decreasing the electronic transport capacity and thus increasing ROS generation (Pessayre et al., 2001).

The alterations of the function and activity of these metabolically relevant proteins can happen by several means. One way to achieve metabolic regulation by protein function alteration is the regulation of the acetylation. Sirtuin 3 (SirT3) is localized within the mitochondrial 
matrix, where it controls acetylation levels of enzymes involved in energy metabolism processes, including the respiratory chain, Krebs cycle and mitochondrial fatty acid $\beta$-oxidation. High-fat diets have been shown to reduce SirT3 activity, causing hyperacetylation of a diverse set of liver proteins, contributing to reduced efficiency of the mitochondrial oxidative phosphorylation (OXPHOS) and potentiating lipotoxicity under conditions of nutrient excess (Choudhury et al., 2011; Hirschey et al., 2010; Kendrick et al., 2011).

Berberine (BBR) is an isoquinoline alkaloid present in several plants used in traditional Chinese medicine, which is known to reduce blood glucose levels in T2D (Yin et al., 2008). As such, it is considered as a natural product with hypoglycemic action and a potent reducer of bodyweight in obese animals. The action of this compound is associated with a decreased expression of genes involved in lipogenesis, and an increased activity of AMP-activated protein kinase (AMPK) and expression of genes involved in energy expenditure (Gomes et al., 2012; Lee et al., 2006; Zhou et al., 2008). Previous studies demonstrated that BBR might improve insulin sensitivity through a mechanism related to the deactivation of acetyl-CoA carboxylase (ACC) and, thus, increase the rate of fatty acid oxidation (Kong et al., 2009; Lee et al., 2006; Zhang et al., 2009). It was also reported that BBR, in the liver of diabetic rats, decreased fasting glucose, hepatic steatosis and the expression of several gluconeogenic genes such as Phosphoenolpyruvate Carboxykinase (PEPCK) and Glucose-6-Phosphatase (G6Pase) (Xia et al., 2011).

To this day, to the best of our knowledge, no study has fully characterized hepatic mitochondrial function when exposed to BBR. In this study, we evaluated the effects of BBR on the mitochondrial function of steatotic livers and how this protects the liver against the conditions associated with the incidence of obesity.

\section{Experimental procedures}

\subsection{Animals, diets and treatments}

Male Sprague-Dawley rats aged 6-8 weeks were housed under controlled light and humidity conditions and given ad libitum access to water and standard chow (control group) or an isocaloric high fat diet (HFD) for 12 weeks. After 12 weeks, the HFD group was divided into two groups: one was kept in the same conditions and a third group was maintained on HFD with a supplement of BBR $(100 \mathrm{mg} / \mathrm{kg} / \mathrm{day})$ in the drinking water for 4 more weeks (BBR). After these 16 weeks of study, animals were sacrificed, in the fed state, by cervical dislocation, in the early hours of the morning.

BBR and food intake were monitored over the study to insure that each animal consumed roughly the same amount of BBR and calories (by water volume consumption and food weight in cage). The diets were purchased from Research Diets, Inc (New Jersey, USA). The diet used to induce obesity (HFD) has $60 \%$ of its calorie count derived from fat, whereas the control diet $(\mathrm{Ctl})$ has $10 \%$ calories derived from fat. All experimental procedures respected the guidelines of the European Directive 10386/609/CEE, the Declaration of Helsinki and those present in the Portuguese law.

\subsection{Materials}

Except when noted, all compounds were purchased from Sigma Chemical Co. (St. Louis, MO). All reagents and chemicals used were of the highest grade of purity commercially available.

\subsection{Plasma biochemical determination}

Plasma samples were immediately collected after animal sacrifice and enzymatic determinations were performed using commercial kits. Plasma glucose was determined by a glucometer (Accu-chek Aviva Roche, Portugal). Plasma insulin levels were determined by ELISA (EZRMI-13K - Millipore, Portugal). Plasma leptin and adiponectin levels were measured using a commercially available kit (Invitrogen, Spain). Triglycerides were extracted using isopropanol and quantified using a colorimetric based commercially available kit (Cromatest, Spain). All other plasma measurements were conducted by the Laboratório de Análises Clínicas da Faculdade de Farmácia da Universidade de Coimbra. To determine insulin sensitivity and glucose tolerance a glucose tolerance test was performed, by oral gavage of 1.5 g glucose/kg body weight. Blood samples were taken from the tail vein at several time points after the bolus injection and immediately flash-frozen for later analysis.

\subsection{FAME analysis}

Fatty acid composition was determined by gas-liquid chromatography after trans-methylation, as previously described (Peixoto et al., 2004). Before fatty acid derivatization, the exogenous heptadecanoic acid (C:17) was added to the lipid extract as an internal standard. Hepatocyte fatty acid methyl esters (FAMEs) were analyzed with a Trace GC 2000 (Thermo-Finnigan, Thermo Electron Corporation, Austin, TX) with helium as carrier gas, using an Agilent J \&W Scientific (Agilent Technologies, Inc., Santa Clara, CA) capillary column (SUPELCOWAX 10, $30 \mathrm{~m}, 0.25 \mathrm{~mm}$ id., $0.5 \mu \mathrm{m}$ film thickness) and a temperature program as follows: $2 \mathrm{~min}$ at $140{ }^{\circ} \mathrm{C}, 4^{\circ} \mathrm{C} \mathrm{min}{ }^{-1}$ to $220{ }^{\circ} \mathrm{C}$ and $10 \mathrm{~min}$ at the upper temperature. Injector and flame ionization detector (FID) temperatures were kept at 250 and $270{ }^{\circ} \mathrm{C}$, respectively. Samples of $4 \mu \mathrm{L}$ were split-injected and solvent blanks were periodically checked. FAMEs were identified by comparison with the retention time of FAME standard (37-Component FAME Mix).

\subsection{Preparation of liver mitochondria}

Mitochondria were isolated from animals of the three experimental groups by differential centrifugation, as previously described (Gazotti et al., 1979), with modifications (Palmeira et al., 1994). The homogenization buffer contained $250 \mathrm{mM}$ sucrose, $10 \mathrm{mM}$ HEPES (pH 7.4), $0.5 \mathrm{mM} \mathrm{EGTA}$ and $0.1 \%$ fat-free bovine serum albumin. EGTA and bovine serum albumin were omitted from the final washing medium, adjusted at pH 7.4. Briefly, after homogenization of the minced blood-free hepatic tissue, the homogenate was centrifuged at $2500 \times \mathrm{g}$ for $10 \mathrm{~min}$ at $4{ }^{\circ} \mathrm{C}$. The resulting supernatant was spun at $10,000 \times g$ for $10 \mathrm{~min}$ at $4{ }^{\circ} \mathrm{C}$ to pellet mitochondria, which were resuspended in more washing medium. This last step was repeated two more times to obtain an ultrapure mitochondrial fraction. Protein content was determined by the biuret method (Gornall et al., 1949) calibrated with bovine serum albumin.

\subsection{Mitochondrial oxygen consumption}

Oxygen consumption of isolated mitochondria was polarographically determined with a Clark oxygen electrode (Estabrook, 1967), as previously described (Rolo et al., 2000). Mitochondria (1 mg) were suspended under constant magnetic stirring, at $25^{\circ} \mathrm{C}$, in $1.4 \mathrm{~mL}$ of standard respiratory medium ( $130 \mathrm{mM}$ sucrose, $50 \mathrm{mM} \mathrm{KCl}, 5 \mathrm{mM} \mathrm{MgCl}$, $5 \mathrm{mM} \mathrm{KH}_{2} \mathrm{PO}_{4}, 50 \mu \mathrm{M}$ EDTA, and $5 \mathrm{mM}$ HEPES (pH 7.4) and $2 \mu \mathrm{M}$ rotenone). Mitochondria were energized with succinate $(5 \mathrm{mM})$ and State 3 respiration was induced by adding $200 \mathrm{nmol}$ ADP. The oxygen consumption was also measured in the presence of $1 \mu \mathrm{M}$ carbonylcyanideP-trifluoromethoxyphenylhydrazon (FCCP). State 3 and Respiratory Control Ratio (RCR) were calculated according to Chance and Williams (1956).

\subsection{Membrane potential $(\Delta \Psi)$ measurements}

$\Delta \Psi$ was estimated using an ion-selective electrode to measure the distribution of tetraphenylphosphonium $\left(\mathrm{TPP}^{+}\right)$according to previously established methods (Kamo et al., 1979; Palmeira et al., 1994). The 
voltage response of the $\mathrm{TPP}^{+}$electrode to $\log \left[\mathrm{TPP}^{+}\right]$was linear with a slope of $59 \pm 1$, in conformity with the Nernst equation. Reactions were carried out at $25^{\circ} \mathrm{C}$, in a temperature controlled water-jacketed chamber with magnetic stirring. Mitochondria (1 mg) were suspended in $1 \mathrm{~mL}$ of standard respiratory medium (as above) supplemented with $3 \mu \mathrm{M} \mathrm{TPP}^{+}$. Mitochondria were energized with the addition of succinate (5 mM). A matrix volume of $1.1 \mu \mathrm{L} / \mathrm{mg}$ protein was assumed.

\subsection{Enzymatic activities}

Succinate Dehydrogenase activity was polarographically determined as previously described (Singer, 1974). The reaction was carried out at $25{ }^{\circ} \mathrm{C}$ in $1.4 \mathrm{~mL}$ of standard respiratory medium (as in mitochondrial respiration) supplemented with $5 \mathrm{mM}$ succinate, $2 \mu \mathrm{M}$ rotenone, $0.1 \mu \mathrm{g}$ Antimycin A, $1 \mathrm{mM} \mathrm{KCN}$ and $0.3 \mathrm{mg}$ Triton X-100. After the addition of freeze-thawed mitochondria $(0.25 \mathrm{mg})$ the reaction was initiated with $1 \mathrm{mM}$ phenazine methosulfate (PMS).

Cytochrome c Oxidase activity was polarographically determined, as previously described (Brautigan et al., 1978). The reaction was carried out at $25^{\circ} \mathrm{C}$ in $1.4 \mathrm{~mL}$ of standard respiratory medium supplemented with $2 \mu \mathrm{M}$ rotenone, $10 \mu \mathrm{M}$ oxidized Cytochrome $c, 0.3 \mathrm{mg}$ Triton $\mathrm{X}-100$ and freeze-thawed mitochondria $(0.25 \mathrm{mg})$, after which the reaction was initiated by adding $5 \mathrm{mM}$ ascorbate plus $0.25 \mathrm{mM}$ tetra methylphenylene-diamine (TMPD).

ATPase activity was determined spectrophotometrically at $660 \mathrm{~nm}$, in association with ATP hydrolysis, as previously described (Teodoro et al., 2008). Briefly, the reaction was carried out at $37{ }^{\circ} \mathrm{C}$, in $2 \mathrm{~mL}$ reaction medium (125 mM sucrose, $65 \mathrm{mM} \mathrm{KCl,} 2.5 \mathrm{mM} \mathrm{MgCl}$ and $0.5 \mathrm{mM}$ HEPES, $\mathrm{pH}$ 7.4). After the addition of freeze-thawed mitochondria $(0.25 \mathrm{mg})$ the reaction was initiated with the addition of $2 \mathrm{mM} \mathrm{Mg}^{2+}$-ATP, in the presence or absence of oligomycin $(1 \mu \mathrm{g} / \mathrm{mg}$ protein). After $10 \mathrm{~min}$, the reaction was stopped by adding $1 \mathrm{~mL}$ of $40 \%$ trichloroacetic acid and the samples were centrifuged for $5 \mathrm{~min}$ at $3000 \mathrm{rpm}$. $2 \mathrm{~mL}$ of ammonium molybdate plus $2 \mathrm{~mL} \mathrm{H}_{2} \mathrm{O}$ was then added to $1 \mathrm{~mL}$ of supernatant. ATPase activity was calculated as the difference in total absorbance and absorbance in the presence of oligomycin.

\subsection{ATP quantification}

Adenosine nucleotide extraction was performed as follows. Tissue samples $(20 \mathrm{mg}$ ) were pulverized with a mortar and pestle in liquid $\mathrm{N}_{2}$ and homogenized in ice-cold $25 \mu \mathrm{L}$ of $\mathrm{KOH}$ buffer (KOH $2.5 \mathrm{M}$, $\mathrm{K}_{2} \mathrm{HPO}_{4} 1.5 \mathrm{M}$ ). Homogenates were vortexed and centrifuged at $14,000 \times g$ for $2 \mathrm{~min}$, at $4{ }^{\circ} \mathrm{C}$. The supernatants were collected and dissolved in $100 \mu \mathrm{L}$ of $\mathrm{K}_{2} \mathrm{HPO}_{4} 1 \mathrm{M}$. pH was adjusted to 7 and samples were frozen at $-80{ }^{\circ} \mathrm{C}$ for posterior use. On assay day, adenosine nucleotides were quantified with an ATP bioluminescent assay kit (Sigma-Aldrich) on a Victor ${ }^{3}$ (PerkinElmer, Waltham, MA) plate reader.

\subsection{Evaluation of reactive oxygen species (ROS) generation}

ROS generation was fluorometrically determined using a PerkinElmer VICTOR ${ }^{3}$ plate-reader, with an excitation wavelength of $485 \mathrm{~nm}$ and an emission wavelength of $538 \mathrm{~nm}$, respectively corresponding to the excitation and emission wavelengths of $2^{\prime}, 7^{\prime}$ dichlorodihydrofluorescein diacetate ( $\mathrm{H}_{2} \mathrm{DCFDA}$ ) (Zhou et al., 2001). Isolated mitochondria $(1 \mathrm{mg} / \mathrm{mL})$ were suspended in standard respiratory medium and loaded with succinate $5 \mathrm{mM}$ and $\mathrm{H}_{2}$ DCFDA $50 \mu \mathrm{M}$ (prepared in DMSO) for $15 \mathrm{~min}$ at $25^{\circ} \mathrm{C} .200 \mu \mathrm{L}$ of the mitochondrial suspension was loaded into a 96 -well plate and the fluorescence was monitored for $30 \mathrm{~min}$ to calculate the rate of ROS formation. The results were expressed as arbitrary relative fluorescence units (RFUs).

\subsection{Measurement of the mitochondrial permeability transition}

Mitochondrial swelling was estimated by changes in light scattering, as monitored spectrophotometrically at $540 \mathrm{~nm}$ (Palmeira and Wallace, 1997). Reactions were carried out at $25{ }^{\circ} \mathrm{C}$. Recording was started by the addition of mitochondria ( $1 \mathrm{mg}$ ) to $2 \mathrm{~mL}$ of swelling medium $(200 \mathrm{mM}$

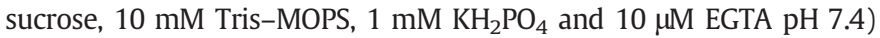
supplemented with $3 \mu \mathrm{M}$ rotenone and $5 \mathrm{mM}$ succinate. After a brief period for the recording of basal absorbance, different amounts of $\mathrm{Ca}^{2+}$ were added and the resulting alterations in light scattering were registered.

\subsection{Western blotting analysis}

Tissue homogenates were lysed in ice-cold RIPA lysis buffer supplemented with a cocktail of protease and phosphatase inhibitors (Sigma-Aldrich). Equal amounts of protein (measured with a Bicinchoninic Acid Kit, Sigma-Aldrich) were loaded and electrophoresed on a home-made SDS-polyacrylamide gel and transferred to a polyvinylidene difluoride membrane (Bio-Rad Laboratories, Hercules, CA). Membranes were blocked with 5\% blocking solution (Bio-Rad) for $2 \mathrm{~h}$ and incubated in Tris-buffered saline (TBS) supplemented with $1 \%$ Tween-20 (TBS-T) and blocking solution $0.5 \%$, overnight at $4{ }^{\circ} \mathrm{C}$, with anti-PGC-1 $\alpha$ (Cell Signaling Technology, Beverly, MA, 1:100), anti-SirT1 (Cell Signaling Technology, 1:1000), anti-SirT3 (Cell Signaling Technology, 1:500), anti-TFAM (Aviva Systems Biology, San Diego, CA, 1:2000), anti-NRF1 (Aviva Systems Biology, 1:1000), anti-ATP synthase (Aviva Systems Biology, 1:1000), anti-COX I (MitoSciences, Eugene, OR, 1:1000), anti-COX IV (MitoSciences, 1:10,000) or anti- $\beta$-actin (SigmaAldrich, 1:5000) antibody. The following day, membranes were washed in room temperature TBS-T 3 times for 30 min and incubated with a corresponding secondary antibody (Invitrogen, Carlsbad, CA). Membranes were then washed in room temperature TBS-T 3 times for $15 \mathrm{~min}$ and immunodetection was performed with WesternDot 625 goat antirabbit or goat anti-mouse western blot kits (Invitrogen). Membranes were imaged using a VersaDoc Instrument (Bio-Rad).

\subsection{RNA isolation and genetic expression evaluation by $q P C R$}

Flash-frozen liver RNA was extracted with an AxyPrep RNA extraction kit (Axygen, Union City, CA). Total RNA was quantified by the use of the Experion Automated Electrophoresis Station (Bio-Rad) and cDNA was generated from RNA with an iScript cDNA synthesis kit (Bio-Rad), following manufacturer's recommendations. Semi-quantitative realtime PCR was conducted with a SYBR Green real-time PCR kit (BioRad), following manufacturer's recommendations. Utilized primers were, 5'-3' (Gene, Forward primer, Reverse primer.): COX II, tgg ctt aca aga cgc cac at; tgg geg tct att gtg ctt gt; COX IV, act acc cct tgc ctg atg tg, act cat tgg tgc cct tgt tc; PDK4, cct ttg gct ggt ttt ggt ta, cac cag tca tca gcc tca ga; PEPCK, ccc agg agt cac cat cac tt, ttc gta gac aag ggg gac ac; atg tgt cgc ctt ctt gct ct, atc tac tgc ctg ggg acc tt; SirT1, cca gat cct caa gcc atg tt, gat cct ttg gat tcc tgc aa; SirT3, gct gcc agc aag gtt ctt ac, cct ttc cac acc ctg gac ta, TFAM, atc aag act gtg cgt gca tc, aaa gcc cgg aag gtt ctt ag; and 18S, cgc ggt tct att ttg ttg gt, agt cgg cat cgt tta $\operatorname{tgg}$ tc.

\subsection{SirT3 activity}

SirT3 activity was measured in mitochondrial extracts using the SirT3 direct fluorescent screening assay kit (Cayman Chemical Company, Ann Arbor, MI). A kit-supplied acetylated, fluorescently labeled sirtuin-3 peptide substrate is mixed with the freeze-thawed mitochondrial preparations isolated before $\left(25 \mathrm{mg}\right.$ ) at $37{ }^{\circ} \mathrm{C}$ for $45 \mathrm{~min} .25 \mu \mathrm{L}$ of developer is then added and following $30 \mathrm{~min}$ of incubation, the fluorescence intensity was measured on a Victor ${ }^{3}$ plate reader, with excitation and emission wavelengths of 340 and $440 \mathrm{~nm}$, respectively. It is 
noteworthy that this kit might detect the activity of other deacetylating sirtuins, but since in mitochondria only SirT3-5 are present and since SirT4 is not a deacetylase (Huang et al., 2010) and SirT5 has an extremely reduced deacetylating activity (Du et al., 2011), it is safe to assume that SirT3 is accounted for virtually all of the fluorescence.

\subsection{Statistical analysis}

Data is represented as a mean of $\mathrm{n} \geq 4 \pm$ SEM, and statistical significance was determined using the one-way (two-way for the swelling analysis) ANOVA test with a Bonferroni correction (GraphPad Prism software, La Jolla, CA). A value of $p<0.05$ was considered statistically significant.

\section{Results}

\subsection{Animal model characterization}

In order to demonstrate the effectiveness of the high-fat diet in terms of body weight gain, the animals were weighted once a week, with the main results being summarized below. The high fat feeding unsurprisingly caused the body weight of the animals to increase significantly. In fact, just after one month of high fat feeding, the body weight increase of the HFD group was statistically significant. BBR treatment completely reversed the effect of high-fat feeding during 4 months in just one month (Fig. 1A). There were no effects detected on food consumption in any of the diets by simple evaluation of the weight of diets placed on the cages (data not shown).

Following total body weight, the total liver weight in HFD was also significantly elevated; again, BBR prevented this phenotype (Ctl: $12.6 \mathrm{~g} \pm 0.4$; HFD: $17.14 \mathrm{~g} \pm 1.3$; BBR: $10.86 \mathrm{~g} \pm 0.7$ - HFD liver weight is statistically heavier than $\mathrm{Ctl}$ and $\mathrm{BBR})$. As far as blood serum biochemical parameters, several alterations were found, induced by the treatments (Table 1). Of particular relevance, BBR reverted the high-fat diet effects on fasting and fed glycemic levels, HbA1c, circulating triglycerides and phospholipids, leptin and insulin levels (Table 1). The return of all of these metabolic indicators back to control levels illustrates a positive effect of BBR on reverting the effects of feeding a high-fat diet.

The evaluation of the data from the oral glucose tolerance test demonstrated that the HFD animals were incapable to rapidly reduce their circulating glucose levels, at least as fast as the Ctl animals (Fig. 1B). BBR restored this capacity, which could indicate that BBR recovered the animals' insulin sensitivity. As such, insulin level quantification was required. Data from such assay (Fig. 1C) demonstrates that HFD animals were hyperinsulinemic, while BBR was able to recover insulin sensitivity to the animals.

Moreover, FAME analysis of the spectra of fatty acid methyl esters demonstrates the same pattern: "bad" (saturated or trans) fats (Stearic, Elaidic and Linolelaidic acids) are elevated in HFD animals, while "good" (unsaturated) fats (Oleic, Linolenic and Eicosatrienoic acids) are elevated in BBR-supplemented animals (Fig. 2).

\subsection{Effects of high fat feeding and berberine supplementation on liver mitochondrial function}

Since mitochondria are the main site for energy generation in the eukaryotic cell (as well as being crucial for a panoply of other biological processes), we evaluated how high fat feeding and BBR supplementation affected mitochondrial function. Oxidative phosphorylation relies on the generation of a transmembrane electrochemical potential (in the form of a protonic gradient) or $\Delta \Psi$, associated with molecular oxygen consumption. These parameters can be directly measured in isolated mitochondria. No significant variation on the activity of citrate
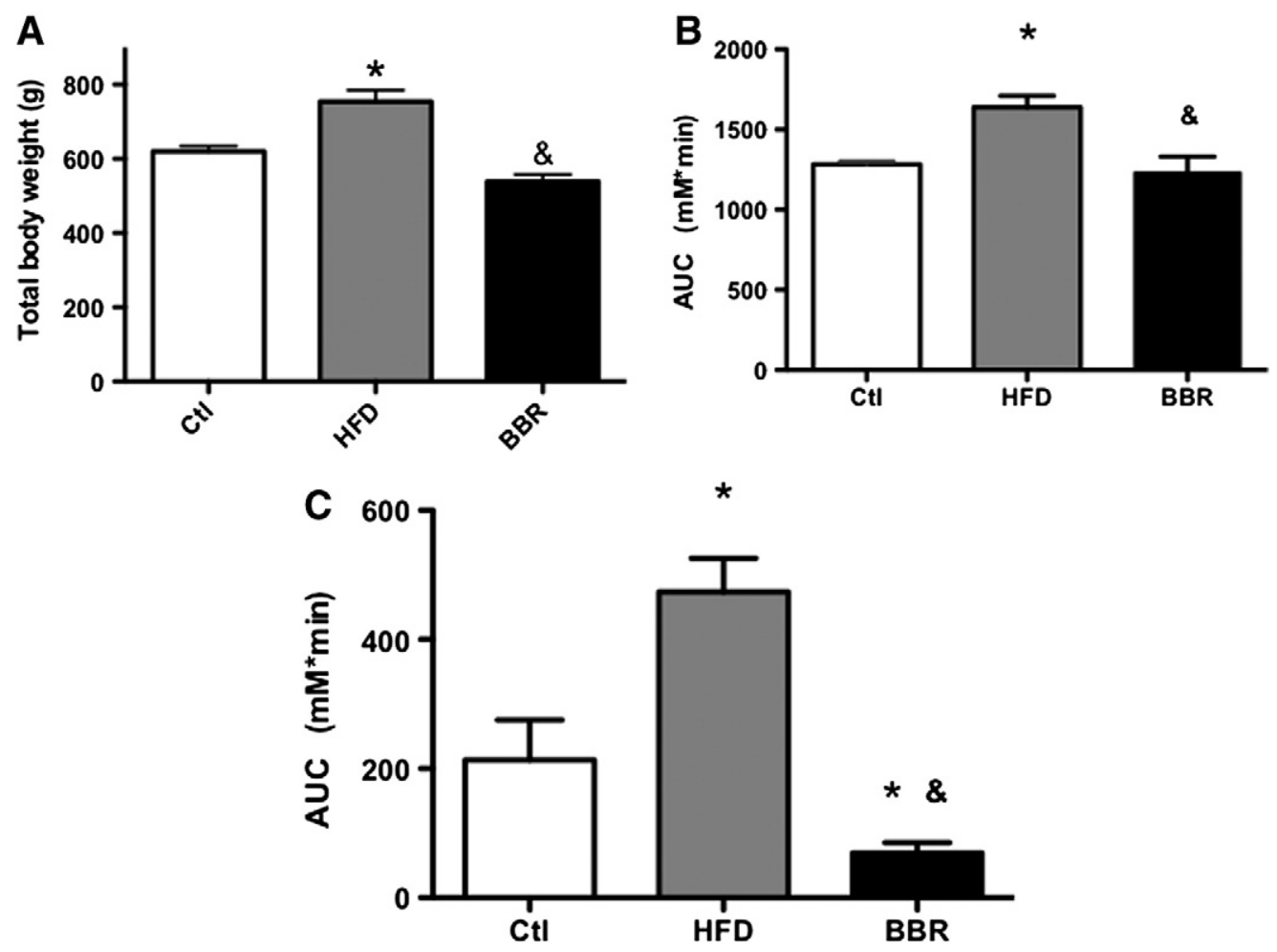

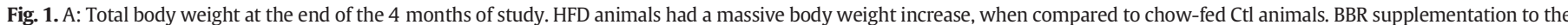

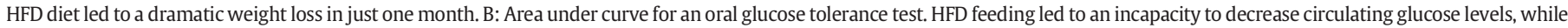

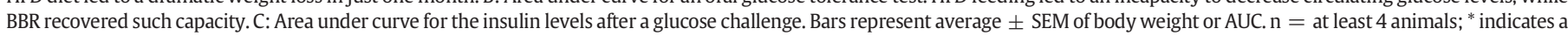
statistically significant difference versus control $(p<0.05)$; \& indicates a statistically significant difference versus HFD $(p<0.05)$. 
Table 1

Blood serum biochemical parameters. HFD animals had alterations in several biochemical parameters, most of them were reversed by BBR supplementation.

\begin{tabular}{|c|c|c|c|}
\hline & Ctl & HFD & BBR \\
\hline $\begin{array}{l}\text { Fed } \\
\text { Glycemia (mg/dL) }\end{array}$ & $130.6 \pm 5.48$ & $157.1 \pm 9.43^{\mathrm{a}}$ & $114.8 \pm 4.00^{\mathrm{b}}$ \\
\hline $\begin{array}{l}\text { Fasted } \\
\quad \text { Glycemia }(\mathrm{mg} / \mathrm{dL})\end{array}$ & $67.7 \pm 2.64$ & $79.08 \pm 3.20^{\mathrm{a}}$ & $63.14 \pm 4.36^{\mathrm{b}}$ \\
\hline Insulin (pmol/L) & $90.25 \pm 8.99$ & $159.0 \pm 26.76^{\mathrm{a}}$ & $67.25 \pm 8.37^{\mathrm{b}}$ \\
\hline $\mathrm{HbA1c}(\%)$ & $3.28 \pm 0.06$ & $3.57 \pm 0.06^{\mathrm{a}}$ & $3.30 \pm 0.05^{\mathrm{b}}$ \\
\hline HDL cholesterol (mg/dL) & $39.7 \pm 3.87$ & $63.00 \pm 5.59^{\mathrm{a}}$ & $39.33 \pm 4.40^{\mathrm{b}}$ \\
\hline $\begin{array}{l}\text { Non-HDL cholesterol } \\
(\mathrm{mg} / \mathrm{dL})\end{array}$ & $27.4 \pm 1.82$ & $36.0 \pm 3.94$ & $28.7 \pm 4.54$ \\
\hline Free fatty acids (mM) & $0.46 \pm 0.06$ & $0.40 \pm 0.07$ & $0.54 \pm 0.03$ \\
\hline Leptin $(\mathrm{pg} / \mathrm{mL})$ & $4434 \pm 714$ & $20,399 \pm 3869^{a}$ & $579 \pm 93.1^{\mathrm{b}}$ \\
\hline Adiponectin (ng/mL) & $10,407 \pm 1727$ & $19,111 \pm 1615^{a}$ & $31,944 \pm 3893^{\mathrm{a}, \mathrm{b}}$ \\
\hline Triglycerides (mg/dL) & $56.0 \pm 6.21$ & $94.9 \pm 9.66^{\mathrm{a}}$ & $44.3 \pm 5.75^{\mathrm{b}}$ \\
\hline Atherogenic index & $1.66 \pm 0.05$ & $1.56 \pm 0.06$ & $1.72 \pm 0.07$ \\
\hline Phospholipids (mg/dL) & $115.5 \pm 7.88$ & $158.5 \pm 9.40^{\mathrm{a}}$ & $116.8 \pm 7.78^{\mathrm{b}}$ \\
\hline Albumin $(\mathrm{mg} / \mathrm{dL})$ & $3.30 \pm 0.05$ & $3.25 \pm 0.05$ & $3.07 \pm 0.06^{\mathrm{a}}$ \\
\hline Creatinine $(\mathrm{mg} / \mathrm{dL})$ & $0.52 \pm 0.01$ & $0.55 \pm 0.02$ & $0.52 \pm 0.02$ \\
\hline Ureic nitrogen (mg/dL) & $15.6 \pm 1.24$ & $11.9 \pm 1.48$ & $15.43 \pm 2.77$ \\
\hline Uric acid $(\mathrm{mg} / \mathrm{dL})$ & $1.58 \pm 0.05$ & $1.60 \pm 0.37$ & $1.12 \pm 0.04$ \\
\hline $\begin{array}{l}\text { Alanine aminotransferase } \\
(\mathrm{U} / \mathrm{L})\end{array}$ & $52.4 \pm 1.88$ & $72.0 \pm 9.81$ & $66.0 \pm 4.57$ \\
\hline $\begin{array}{l}\text { Aspartate aminotransferase } \\
\text { (U/L) }\end{array}$ & $157.9 \pm 8.21$ & $154.0 \pm 5.32$ & $157.0 \pm 9.32$ \\
\hline Alkaline phosphatase (U/L) & $147.2 \pm 21.25$ & $270.5 \pm 26.88^{a}$ & $142.7 \pm 34.10^{\mathrm{b}}$ \\
\hline$\gamma$-Glutamyl transferase & $1.11 \pm 0.26$ & $0.75 \pm 0.16$ & $0.50 \pm 0.22$ \\
\hline Total bilirubin (mg/dL) & $0.13 \pm 0.02$ & $0.12 \pm 0.02$ & $0.15 \pm 0.02$ \\
\hline $\begin{array}{l}\text { Lactate dehydrogenase } \\
\quad(\mathrm{U} / \mathrm{L})\end{array}$ & $1157 \pm 158$ & $1460 \pm 541$ & $707 \pm 84.2$ \\
\hline Creatine kinase (U/L) & $8756 \pm 2635$ & $18,462 \pm 7416$ & $6322 \pm 1362$ \\
\hline Acid phosphatase (U/L) & $20.08 \pm 1.27$ & $21.77 \pm 1.05$ & $16.30 \pm 0.93^{\mathrm{a}}$ \\
\hline
\end{tabular}

a Indicates a statistically significant difference versus control $(p<0.05)$.

b Indicates a statistically significant difference versus HFD $(p<0.05)$.

synthase (a mitochondrial enzymatic marker) was found between the different experimental groups (data not shown).

\subsubsection{Mitochondrial membrane potential}

Fig. 3A illustrates the membrane potential of isolated liver mitochondria from the animals of the different experimental groups, when energized with succinate.
Comparative to chow controls, high fat feeding led to a significant decrease in mitochondrial $\Delta \Psi$. However, BBR caused the improvement in mitochondrial coupling, rescuing the high fat effect (Fig. 3A).

ADP-induced depolarization was curiously not affected by the high fat feeding; nonetheless, BBR treatment restored the depolarization potential (Fig. 3A), suggesting an overall beneficial effect on BBR on mitochondria, which is not damage-dependent (as, for example, acting as a scavenger).

A negative effect of high fat feeding on repolarization potential was unveiled, which was again reverted by BBR (Fig. 3A).

Finally, the lag phase (or time necessary to complete a phosphorylative cycle) was increased due to high fat feeding, illustrating a clear impairment of mitochondrial function. Once again, BBR was sufficient to revert such effect (Fig. 3A).

\subsubsection{Mitochondrial respiration}

Oxidative phosphorylation capacity of mitochondria isolated from control, high fat fed and BBR-exposed animals was investigated by following oxygen consumption upon succinate oxidation. Mitochondrial respiratory State 3 (consumption of oxygen in the presence of substrate and ADP) was decreased in mitochondria isolated from HFD animals, an effect reversed by BBR (Fig. 3B). Similarly, oxygen consumption in the presence of FCCP, a well-known respiratory chain uncoupler, was also decreased in HFD and, once again, this effect was absent when BBR was supplemented to the high fat fed animals (Fig. 3B).

Surprisingly, no effects of high fat feeding or BBR intake were found in either State 4 respiration or in the presence of oligomycin, a known inhibitor of the mitochondrial $\mathrm{F}_{\mathrm{O}}$-ATP synthase (Fig. 3B), which leads to the conclusion that mitochondrial membrane integrity was not affected throughout the experimental groups, nor was the ATP synthase affected by the high fat diet, causing protonic leakage.

Nevertheless, corresponding to a decrease in State 3 respiration, the Respiratory Control Ratio (RCR) was decreased in HFD mitochondria. BBR was once again able to fully recover this effect (Fig. 3B).

The ADP/O ratio, an indicator of oxidative phosphorylation efficiency, was found to be unaltered, independently of the experimental group (Fig. 3B).

\subsection{Mitochondrial enzymatic activities}

This data was thus indicative of damage to the components of the mitochondrial respiratory chain. As such, the evaluation of the activity

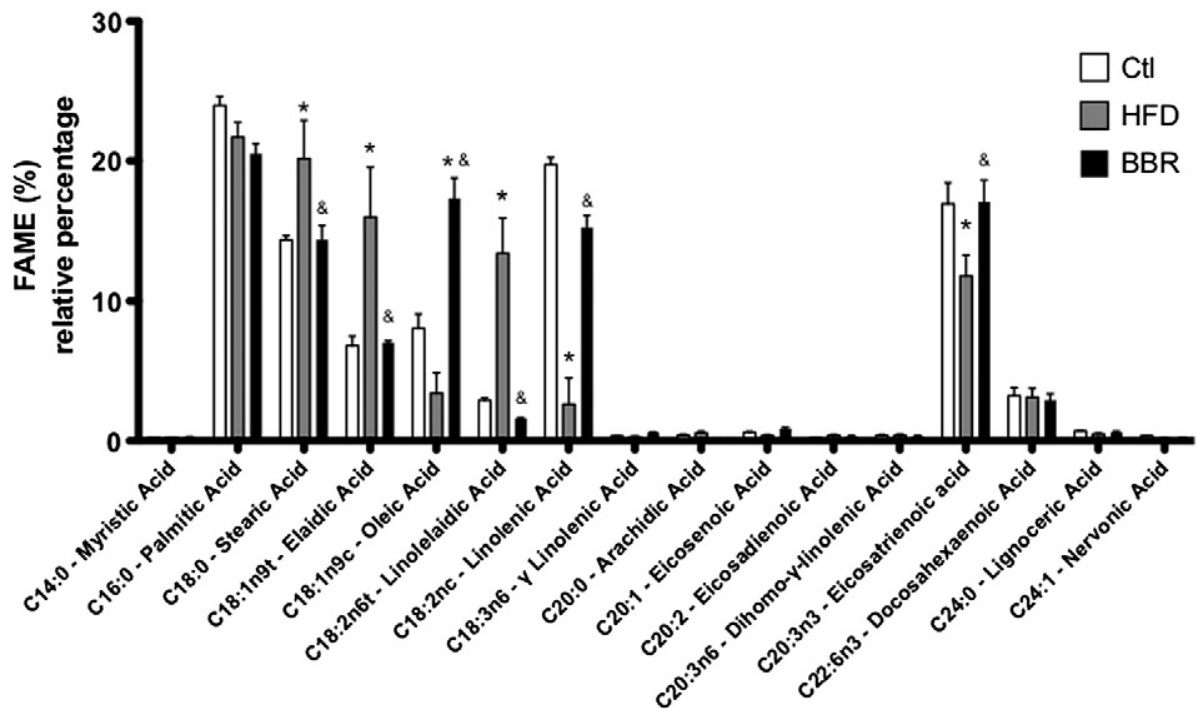

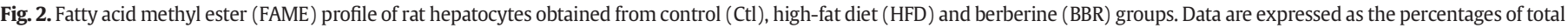

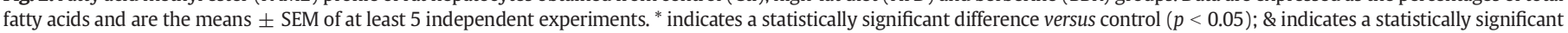
difference versus HFD $(p<0.05)$. 
A

\begin{tabular}{cccc}
\multicolumn{1}{c}{} & Control & HFD & Berberine \\
\cline { 2 - 4 } $\begin{array}{c}\text { Initial Potential } \\
(-m V)\end{array}$ & $216.6 \pm 1.49$ & $208.9 \pm 2.43^{*}$ & $221.3 \pm 1.11^{8}$ \\
$\begin{array}{c}\text { Depolanization } \\
(-m V)\end{array}$ & $27.42 \pm 1.89$ & $25.64 \pm 3.24$ & $37.94 \pm 2.63^{* 8}$ \\
$\begin{array}{c}\text { Repolanization } \\
(-m V)\end{array}$ \\
$\begin{array}{c}\text { Lag phase } \\
(s)\end{array}$ & $213.6 \pm 1.23$ & $203.4 \pm 3.24^{*}$ & $214.8 \pm 0.81^{8}$ \\
& $63.3 \pm 1.92$ & $106.1 \pm 17.70^{\circ}$ & $56.36 \pm 2.72^{8}$
\end{tabular}

B

\begin{tabular}{|c|c|c|c|}
\hline & Control & HFD & Berberine \\
\hline $\begin{array}{l}\text { State 3(nAtoms } \\
\text { O/mg/min) }\end{array}$ & $115.1 \pm 4.36$ & $80.16 \pm 8.38^{\circ}$ & $121.8 \pm 6.17^{*}$ \\
\hline $\begin{array}{l}\text { State } 4 \text { (nAtoms } \\
\text { O/mg/min) }\end{array}$ & $26.91 \pm 2.91$ & $28.95 \pm 3.27$ & $30.83 \pm 2.95$ \\
\hline $\begin{array}{l}\text { Oligomycin (nAtoms } \\
\text { O/mg/min) }\end{array}$ & $14.72 \pm 2.57$ & $16.86 \pm 5.71$ & $17.41 \pm 3.39$ \\
\hline $\begin{array}{l}\text { V FCCP (nAtoms } \\
\text { O/mg/min) }\end{array}$ & $209.7 \pm 11.15$ & $120.1 \pm 12.61^{\circ}$ & $180.7 \pm 12.87^{\circ}$ \\
\hline RCR & $4.78 \pm 0.68$ & $3.04 \pm 0.12^{*}$ & $4.52 \pm 0.45^{*}$ \\
\hline ADP/O & $1.79 \pm 0.03$ & $1.77 \pm 0.05$ & $1.94 \pm 0.17$ \\
\hline
\end{tabular}

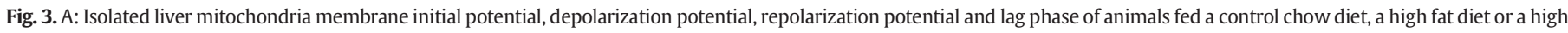

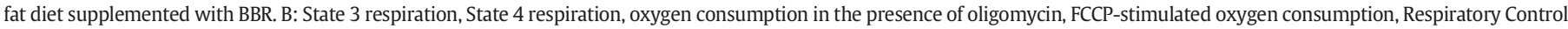

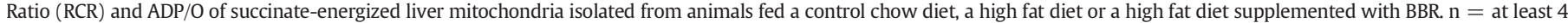
animals; * indicates a statistically significant difference versus control $(p<0.05)$; \& indicates a statistically significant difference versus HFD ( $p<0.05)$.

of both Cytochrome c Oxidase (Complex IV) and Succinate Dehydrogenase (Complex II) revealed an impairment of this activity as a result of high fat feeding. This effect was rescued by the supplementation of BBR (Fig. 4A).

The activity of another element of the mitochondrial oxidative phosphorylation system, the ATP synthase (Complex V), was also evaluated, by resource to a well-known property of the complex, the capacity to reverse its activity when the membrane potential is destroyed, consuming ATP to eject protons, as described in Section 2.8 and before (Teodoro et al., 2008). Once again, BBR rescued the activity of this mitochondrial complex (Fig. 4A). Adding to this, ATP quantification demonstrated that HFD caused a significant decrease in ATP content in HFD animals, an effect that was rescued by BBR (Fig. 4B).

\subsection{Mitochondrial calcium metabolism}

Mitochondrial calcium retention capacity is an important component of mitochondrial cellular activity, for the mitochondria serve as
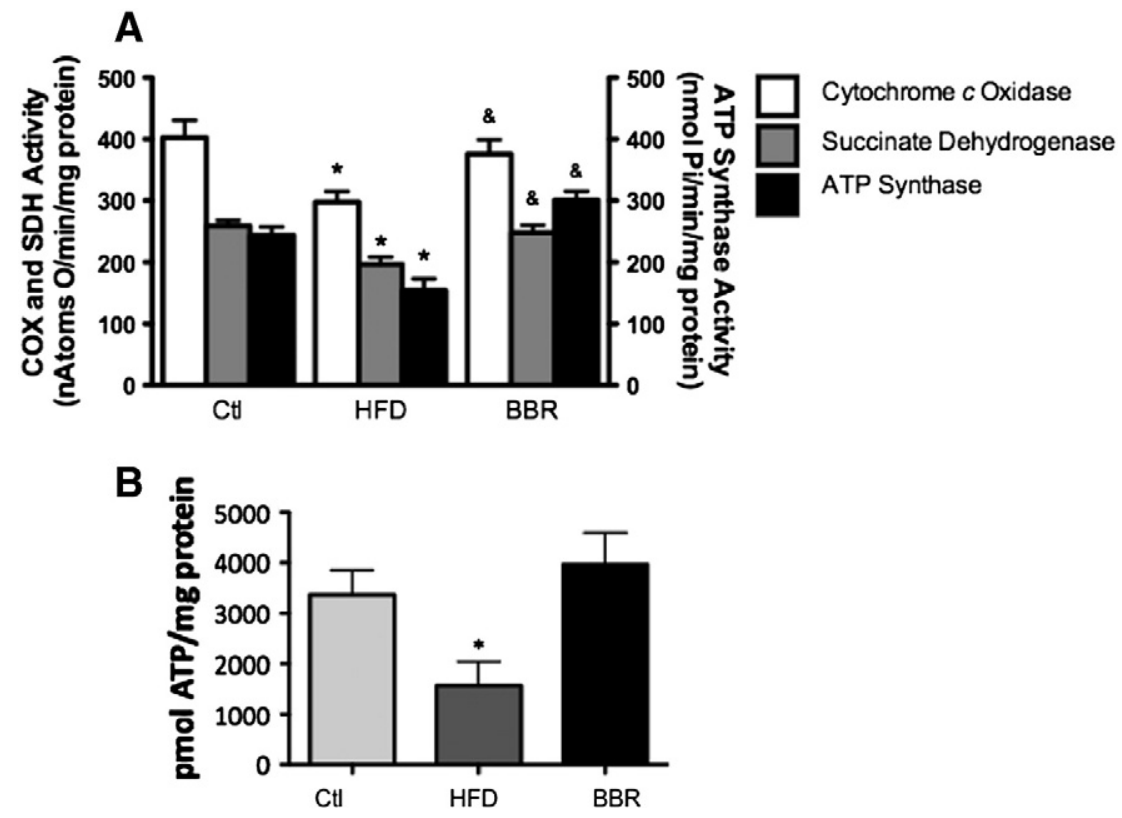

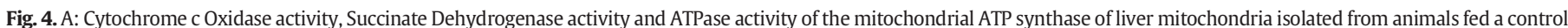

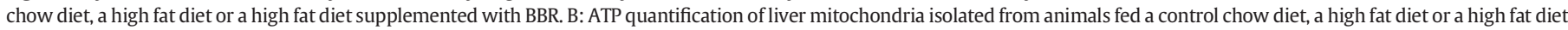

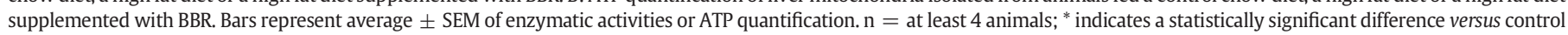
$(p<0.05)$; \& indicates a statistically significant difference versus HFD $(p<0.05)$. 
an intracellular storage site for this secondary messenger and enzymatic cofactor. But this capacity is not limitless for, after a certain threshold, there is induction of the so-called mitochondrial permeability transition (mPT), a phenomenon known for the release of high molecular weight solutes from within the mitochondria, possibly through the formation of a multi-channel pore. Simply put, the amount of calcium the mitochondria can accumulate and for how long is an extremely useful indicator of mitochondrial function and status.

As is visible in Fig. 5A, unstimulated mitochondria do not swell, independently of the diet fed to the animals, as well as mitochondria in the presence of a high calcium dose and Cyclosporine A. This is an indicator

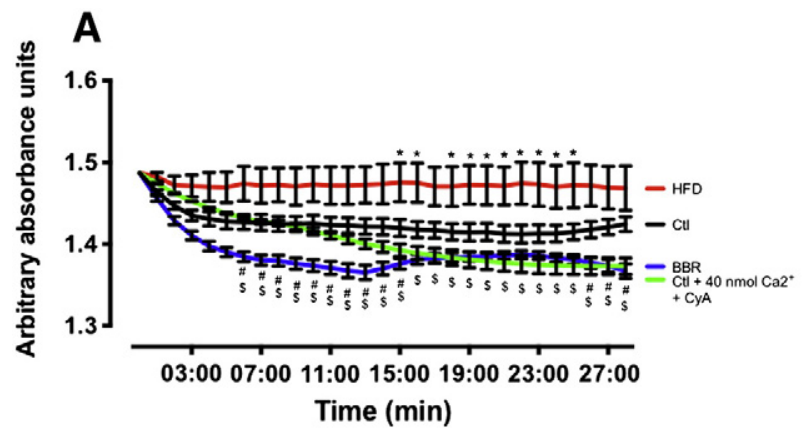

B

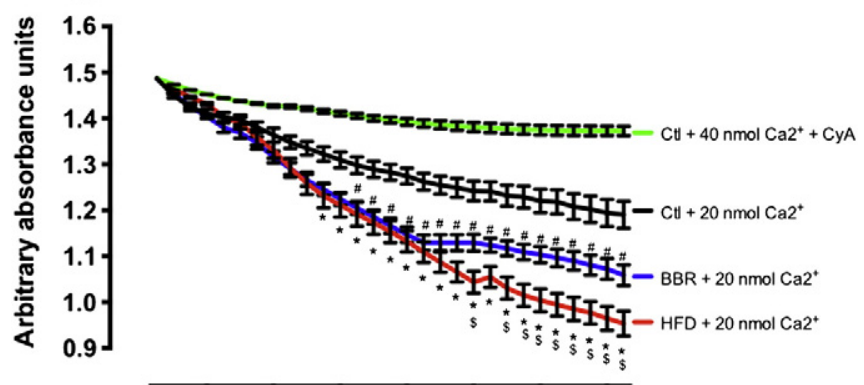

03:00 07:00 11:00 15:00 19:00 23:00 27:00 Time (min)

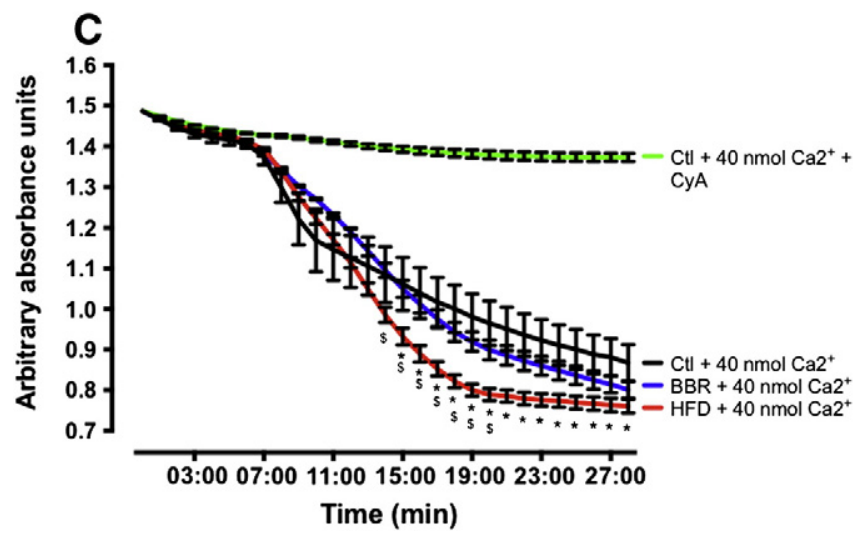

Fig. 5. Mitochondrial permeability transition induction evaluation of liver mitochondria isolated from animals fed a control chow diet, a high fat diet or a high fat diet supplemented with BBR. After a small period of time to obtain a basal absorbance level, 2 concentrations of calcium ( 20 and $40 \mu \mathrm{M}$ ) were added to individual preparations. The absorbance traces were normalized against a control preparation supplemented with calcium and Cyclosporine A, a known mPT inhibitor. Traces represent the average \pm SEM of the assays. A: Traces obtained without calcium addition; B: Traces obtained in the presence of $20 \mu \mathrm{M}$ of calcium; C: Traces obtained in the presence of $40 \mu \mathrm{M}$ of calcium. $\mathrm{n}=$ at least 4 animals; * indicates a statistically significant difference of HFD versus $\mathrm{Ct}$ $(p<0.05)$; \# indicates a statistically significant difference of BBR versus $\mathrm{Ctl}(p<0.05) ; \$$ indicates a statistically significant difference of BBR versus HFD $(p<0.05)$. of the integrity of the isolated mitochondria. But, as soon as calcium is added to the preparation, a clear decrease in absorption is notorious. The faster the decrease, the worse the mitochondrial calcium retention capacity. As so, it is perfectly visible that HFD mitochondria are the ones with the worse calcium retention capacity, being able to retain less than half of the calcium the mitochondria can control (Fig. 5B and C). BBR supplementation appears to be able to recover the calcium retention capacity, especially for a low dose of calcium ( $20 \mu \mathrm{M}$, Fig. 5B). In fact, it takes on average 19 min to statistically differentiate the curves from HFD and BBR mitochondria, in the presence of $20 \mu \mathrm{M}$ of calcium (Ctl and HFD are different from min 10; Ctl and BBR are different from min 12). The addition of a higher dose of calcium $(40 \mu \mathrm{M})$ further demonstrates the same pattern (Fig. 5C). Ctl and BBR are not statistically different, in any point of analysis; however, Ctl and HFD are different from min 15 onward, while HFD and BBR are only different from min 14 to min 20, which indicates that this high dose of calcium is almost at the threshold for these mitochondrial preparations.

\subsection{Mitochondrial ROS generation}

Mitochondrial ROS generation was also evaluated by resource to the $\mathrm{H}_{2} \mathrm{O}_{2}$-sensitive probe, $\mathrm{H}_{2}$ DCFDA, as described in Section 2.10. Despite a trend for higher ROS generation in high fat-fed animals, no statistical difference was found between Ctl and HFD, an effect that could be attributable to a rather high data scattering. Conversely, BBR ROS generation is statistically lower than HFD (Fig. 6).

\subsection{Genetic expression and protein content}

The gene expression of some relevant genes that was evaluated by semi-quantitative real-time PCR, as described in Section 2.13, is resumed in Fig. 7A.

Only the BBR animals' SirT3 levels were found to be elevated when compared to the HFD animals. No other differences were found, despite a clear upregulation of PGC- $1 \alpha$ induced by BBR, and reversal effects of BBR in SirT1 and PDK4 genes.

We further assessed if protein levels were altered by the diets. Surprisingly, we found no alterations caused by any of the diets, for the proteins tested (Fig. 7B and C).

\subsection{SirT3 activity}

Since SirT3 is a mitochondrial matrix protein responsible for controlling metabolism, being expressed in situations of metabolic improvement

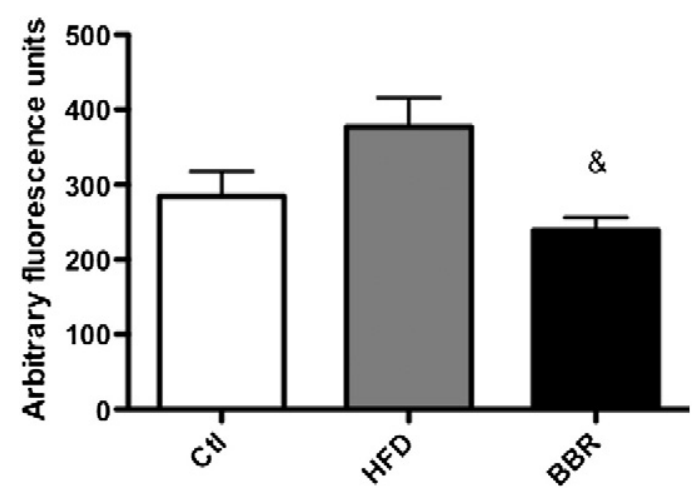

Fig. 6. Mitochondrial ROS generation evaluation of liver mitochondria isolated from animals fed a control chow diet, a high fat diet or a high fat diet supplemented with BBR. After a basal fluorescence measurement, Antimycin A was added to all preparation to block Complex III of the mitochondrial respiratory chain, to induce maximal ROS generation. Bars represent average \pm SEM of Antimycin A-induced ROS generation. $\mathrm{n}=$ at least 4 animals; \& indicates a statistically significant difference versus HFD $(p<0.05)$. 
A

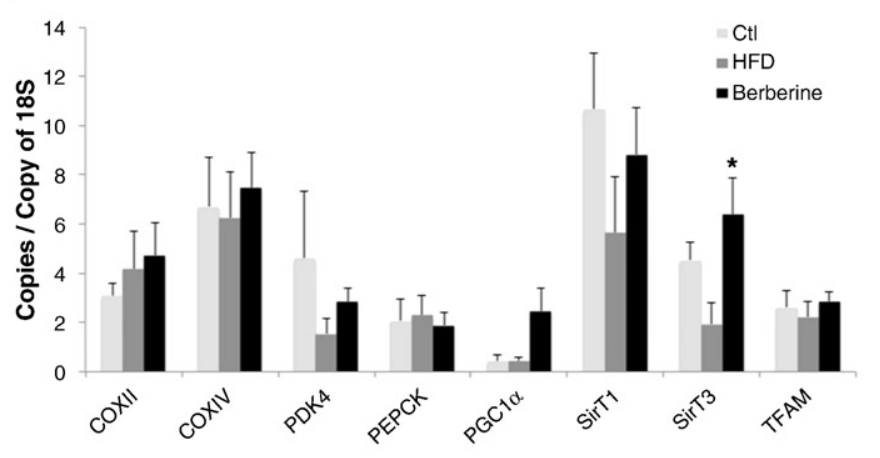

C

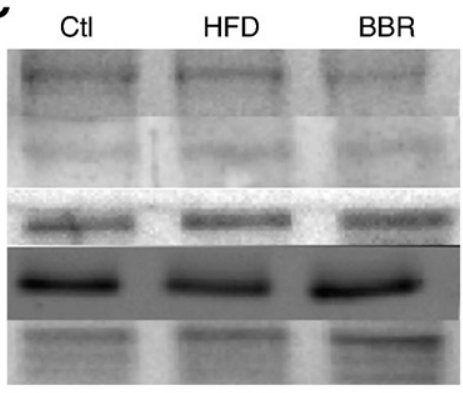

B
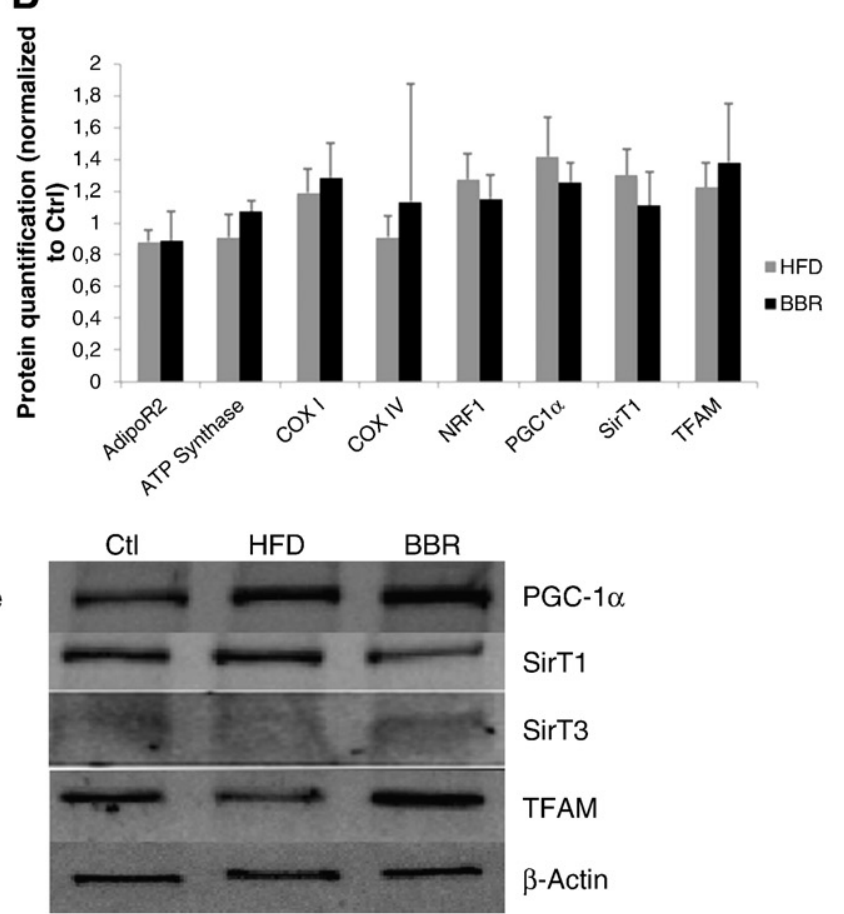

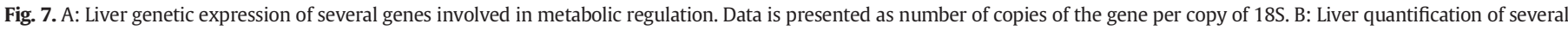

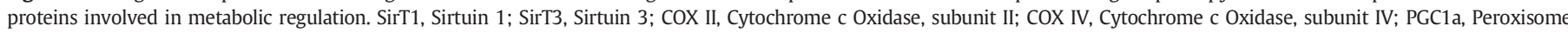

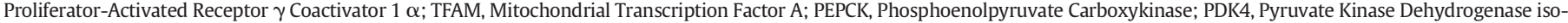

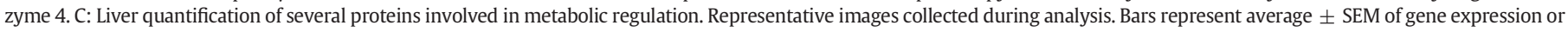
protein quantification. $\mathrm{n}=$ at least 4 animals; ${ }^{*}$ indicates a statistically significant difference versus control $(p<0.05)$.

and fasting (Choudhury et al., 2011), our data suggests that perhaps SirT3 is involved in the effects of BBR supplementation. To analyze this, we evaluated SirT3 activity from the previously obtained mitochondrial preparations, finding that this deacetylase had a decreased activity in HFD animals, which was reversed by BBR supplementation (Fig. 8), which is in accordance with our previous study (Gomes et al., 2012). Since SirT3 is the most capable mitochondrial $\mathrm{NAD}^{+}$-dependent deacetylase ( $\mathrm{Du}$ et al., 2011; Huang et al., 2010) it is clear that this results are virtually dependent on the preparations' SirT3.

\section{Discussion}

Diabetes and its associated conditions, such as obesity and cardiovascular diseases are quickly becoming a leading cause of morbidity

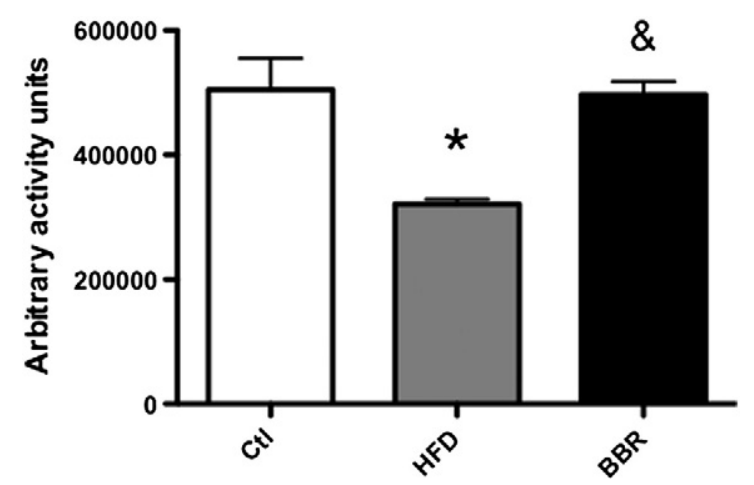

Fig. 8. SirT3 activity of liver mitochondria isolated from animals fed a control chow diet, a high fat diet or a high fat diet supplemented with BBR. Bars represent average \pm SEM of SirT3 activity. $\mathrm{n}=$ at least 4 animals; ${ }^{*}$ indicates a statistically significant difference versus control ( $p<0.05)$; \& indicates a statistically significant difference versus HFD $(p<0.05)$. and mortality in the world. Estimates from the International Diabetes Federation (The Global Burden | International Diabetes Federation, 2013) predict that in the next two decades the number of people afflicted by diabetes will practically double from the current number, to about 438 million, which means that almost $8 \%$ of the world population will be affected. Several works have shown the vital role of glucose homeostasis to prevent or delay the onset of diabetes morbidity. The liver assumes a vital role in the maintenance of adequate circulating glucose levels, by being the main organ for gluconeogenesis and glycogen synthesis (Levine and Haft, 1970). As such, the control of glucose and lipid metabolism in the liver is an attractive target to counteract the pernicious effects of diabetes and its associated complications.

BBR is an isoquinoline alkaloid, present in the roots, bark and other plant parts of several species, as for example, Coptis chinensis and Coptis japonica, Berberis vulgaris, Berberis thunbergii and Berberis aquifolium, Hydrastis canadensis, among others (Domitrovic et al., 2011). These plants are known in traditional eastern medicine for centuries, and are widely used for the treatment of several afflictions, such as intestinal complications and infections. However, the treatment of diabetic patients for diarrhea with these plants (and through them, with their main active compound, BBR) leads to the finding of its hypoglycemic properties. These effects have been extensively explored and investigated in several studies, which demonstrated that BBR is capable of not only improving glycemia in vivo and in vitro (Kim et al., 2007; Leng et al., 2004; Lu et al., 2009; Xia et al., 2011), but also increasing insulin expression, $\beta$-cell regeneration, antioxidant enzyme activity, glucose uptake, while decreasing lipid peroxidation (Wang et al., 2009a; Zhang et al., 2008; Zhou et al., 2009) and cholesterol and triglyceride synthesis (Brusq et al., 2006). All these effects appear to be insulin independent, relying instead in an increase in the AMP/ATP ratio, thus increasing AMPK activity (Cheng et al., 2006; Xia et al., 2011; Zhou et al., 2007). Our previous work has demonstrated that BBR supplementation causes an increase in the expression levels of nicotinamide phosphoribosyltransferase (NAMPT) 
(Gomes et al., 2012), an enzyme that is responsible for $\mathrm{NAD}^{+}$synthesis. As such, despite the above-mentioned reports, not all of BBR effects appear to be mediated through AMPK (Zhou et al., 2010). These previous works support our data, for the BBR-treated animals presented an improved plasma profile (Figs. 1 and 2, Table 1). Of particular interest is the fact that glycemia, phospholipid and triglyceride levels were reverted to (if not overcame) control levels. A notice should be made for adiponectin. As an adipokine, adiponectin is released by adipocytes and its levels seem to be inversely correlated with adiposity and insulin resistance. However, our data demonstrates a different pattern. BBR treated animals demonstrate an increase of circulating adiponectin levels, concomitant with a decrease in body weight and overall regression of obesity-associated characteristics. But adiponectin levels are higher in the HFD animals, when compared with the chow controls, both in total concentration and as an index correlated to body mass (data not shown). This might seem paradoxical, but a previous work demonstrated a dynamic pattern of adiponectin levels in both C57/Bl6 and A/J mice (Bullen et al., 2007), when fed a high-fat diet. Also, an extremely interesting work by Kuda et al. (2009) demonstrated that C57/Bl6 mice, when fed a high fat diet, had a nonstatistical trend towards increased adiponectin levels, when compared to chow-fed controls. We suspect that a similar phenomenon is taking place in our study. Also, when comparing two diets with extremely different fat compositions, the results might not be as predictable, especially when concerning lipid metabolism-involved metabolites. To highlight this idea, leptin levels are predictably elevated in HFD animals, and lowered in BBR. Nevertheless, the massive increase of circulating adiponectin levels in the BBR-treated animals is a clear and consensual indicator of decreased obesity.

Our data unsurprisingly demonstrates that feeding a high fat diet is extremely harmful to liver bioenergetic machinery. Not only did the HFD mitochondria were less efficient, but also took more time to complete a phosphorylative cycle (Fig. 3). This can be attributed to numerous causes, from increased redox imbalance, to membrane alterations, unto protein efficiency alterations (Fig. 4). BBR not only reverted these pernicious effects, but also in some of the evaluated parameters, improved them. As such, it is possible to assume BBR supplementation is hepatoprotective.

Surprisingly, HFD feeding didn't increase ROS generation, despite the fact that BBR was able to reduce it (Fig. 6). Also without a clear positive effect, some works have found that BBR does not have an insulinotropic effect (Wang et al., 2009a, 2011); in fact, one work reported a reduction of insulin secretion after BBR treatment (Zhou et al., 2008). Also, the leptin and adiponectin levels are inconstant in some works, in response to BBR. There is also no consensus in regard to ROS generation. Our work managed to demonstrate an antioxidant effect of BBR, as reported before (Wang et al., 2009b). These are some examples, but these discrepancies might be attributable to different experimental models used and/or different treatment approaches (Wang et al., 2009b).

However, the simple fact that mitochondrial function and activity are completely restored as an effect of BBR is a clear indicator of improvement of hepatic function. Simply by having more efficient mitochondria, hepatocytes are capable of handling more nutrients (such as increased lipids) and thus prevent the harmful effects of their accumulation. By having increased mitochondrial activity, and by the fact that it can induce thermogenesis-involved genes (Lee et al., 2006), BBR can significantly revert and prevent the effects of increased fat intake. As such, BBR utilization has been shown to cause several alterations in genetic expression, specifically in genes coding for metabolic key players. In fact, BBR utilization has been demonstrated to reduce the expression of gluconeogenic and lipogenic genes, while upregulating catabolic genes and some selected transcription factors (Liu et al., 2010; Xia et al., 2011; Zhou and Zhou, 2011). Our results do not demonstrate any alteration, whether on gene expression or protein quantification (for the exception of the expression of SirT3). This might have been caused by the long time course of the study, where these alterations might have occurred long before the animals were sacrificed. Nevertheless, the restoration of the activity of SirT3, a mitochondrial deacetylase might provide an insight into the effects of BBR, for SirT3's activity is related to an improved metabolic profile (Choudhury et al., 2011; Hirschey et al., 2011; Kendrick et al., 2011). The activity of SirT3 as a deacetylase has been demonstrated to be vital for metabolic homeostasis. In fact, the loss of SirT3 has been shown to cause hyperacetylation of mitochondrial proteins, such as Complex I and II, and enzymes involved in mitochondrial lipidic $\beta$-oxidation (Ahn et al., 2008; Cimen et al., 2010; Hirschey et al., 2011), with concomitant decrease in their activity. Furthermore, it has been demonstrated that feeding a high-fat diet causes a SirT3-knockout similar phenotype, through the inhibition of PGC-1 $\alpha$ 's effects on SirT3's expression (Hirschey et al., 2011). Also, SirT3 has been shown to regulate mitochondrial biogenesis, and its knockdown reduced the effects of PGC- $1 \alpha$ on mitochondrial biogenesis (Kong et al., 2010). Since we have previously demonstrated that BBR causes an increase in NAMPT expression, thus leading to an increase in the $\mathrm{NAD}^{+} / \mathrm{NADH}$ ratio (Gomes et al., 2012), it is plausible to assume that the effects of BBR on improvement of hepatic mitochondrial function are vastly dependent on SirT3's increased activity. Several other works have demonstrated that SirT3's activity is the key for metabolic improvement. The sirtuin activator $\mathrm{NAD}^{+}$, generated from its precursor nicotinamide riboside, protects against fatty diet-related injuries (Cantó et al., 2012); but SirT3's activation by calorie restriction and use of lipoic acid provides protection against metabolic pathologies, notably through the improvement of mitochondrial activity (Tauriainen et al., 2011; Valdecantos et al., 2012). As expected, the removal of SirT3 leads to increased metabolic abnormalities and disease (Fernandez-Marcos et al., 2012; Hirschey et al., 2011; Kong et al., 2010).

\subsection{Conclusions}

In conclusion, BBR appears to improve the liver metabolic profile and, to some extent, the whole organism. Mitochondria appear to have a central role mediating the effects of this compound, for the improvement of its parameters will undoubtedly lead to the altered cellular mechanisms that culminate in the reversal of many of the crippling features of constant high fat feeding. Research into development of BBR-like molecules, with similar or even more powerful activity should begin in the near future in order to develop even more powerful antiobesity compounds and therapies. The work presented in this paper demonstrates that BBR can be a powerful anti-obesity and antidiabetic drug in the near future. By showing how BBR leads to improved hepatic mitochondrial function possibly via activation of SirT3, this work further validates BBR (and possible chemically-modified derivatives) as a compound to apply in clinical trials in the next few years.

\section{Acknowledgments}

JST, FVD, APG and ATV were recipients of a Fundação para a Ciência e a Tecnologia PhD scholarship (SFRH/BD/38467/2007, SFRH/BD/38372/ 2007, SFRH/BD/44674/2008 and SFRH/BD/44796/2008, respectively). This project was supported by a FCT grant PTCD/SAU-OSM/72443/ 2006. The authors declare no conflict of interest in the publication of this work.

\section{References}

Ahn, B.-H., Kim, H.-S., Song, S., Lee, I.H., Liu, J., Vassilopoulos, A., Deng, C.-X., Finkel, T., 2008. A role for the mitochondrial deacetylase Sirt3 in regulating energy homeostasis. Proc. Natl. Acad. Sci. U.S.A. 105, 14447-14452.

Brautigan, D.L., Ferguson-Miller, S., Margoliash, E., 1978. Mitochondrial cytochrome c: preparation and activity of native and chemically modified cytochromes c. Meth. Enzymol. 53, 128-164.

Brusq, J., Ancellin, N., Grondin, P., Guillard, R., Martin, S., Saintillan, Y., Issandou, M., 2006. Inhibition of lipid synthesis through activation of AMP kinase: an additional mechanism for the hypolipidemic effects of berberine. J. Lipid Res. 47, 1281. 
Bullen, J.W., Bluher, S., Kelesidis, T., Mantzoros, C.S., 2007. Regulation of adiponectin and its receptors in response to development of diet-induced obesity in mice. Am. J. Physiol. Endocrinol. Metab. 292, E1079-E1086.

Cantó, C., Houtkooper, R.H., Pirinen, E., Youn, D.Y., Oosterveer, M.H., Cen, Y., FernandezMarcos, P.J., Yamamoto, H., Andreux, P.A., Cettour-Rose, P., Gademann, K., Rinsch C., Schoonjans, K., Sauve, A.A., Auwerx, J., 2012. The NAD(+) precursor nicotinamide riboside enhances oxidative metabolism and protects against high-fat diet-induced obesity. Cell Metab. 15, 838-847.

Carley, A.N., Severson, D.L., 2005. Fatty acid metabolism is enhanced in type 2 diabetic hearts. Biochim. Biophys. Acta 1734, 112-126.

Chance, B., Williams, G.R., 1956. The respiratory chain and oxidative phosphorylation. Adv. Enzymol. Relat. Subj. Biochem. 17, 65-134.

Cheng, Z., Pang, T., Gu, M., Gao, A.-H., Xie, C.-M., Li, J.-Y., Nan, F.-J., Li, J., 2006. Berberinestimulated glucose uptake in L6 myotubes involves both AMPK and p38 MAPK. Biochim. Biophys. Acta 1760, 1682-1689.

Choudhury, M., Jonscher, K.R., Friedman, J.E., 2011. Reduced mitochondrial function in obesity-associated fatty liver: SIRT3 takes on the fat. Aging 3, 175-178.

Cimen, H., Han, M.-J., Yang, Y., Tong, Q., Koc, H., Koc, E.C., 2010. Regulation of succinate dehydrogenase activity by SIRT3 in mammalian mitochondria. Biochemistry 49 , 304-311.

Clark, J.M., Diehl, A.M., 2003. Nonalcoholic fatty liver disease. JAMA 289, 3000-3004.

Domitrović, R., Jakovac, H., Blagojević, G., 2011. Hepatoprotective activity of berberine is mediated by inhibition of TNF- $\alpha, \mathrm{COX}-2$, and iNOS expression in $\mathrm{CCl}(4)$-intoxicated mice. Toxicology 280, 33-43.

Du, J., Zhou, Y., Su, X., Yu, J.J., Khan, S., Jiang, H., Kim, J., 2011. Sirt5 is a NAD-dependent protein lysine demalonylase and desuccinylase. Science 334 (6057), 806-809.

Estabrook, R.W., 1967. Mitochondrial respiratory control and the polarographic measurement of ADP:O ratios. Meth. Enzymol. 10, 41-47.

Fernandez-Marcos, P.J., Jeninga, E.H., Canto, C., 2012. Muscle or liver-specific Sirt3 deficiency induces hyperacetylation of mitochondrial proteins without affecting global metabolic homeostasis. Scientific reports.

Gazotti, P., Malmstron, K., Crompton, M., 1979. Preparation and assay of animal mitochondria and submitochondrial particles. In: Carafoli, E., Semenza, G. (Eds.), Membrane Biochemistry: A Laboratory Manual on Transport and Bioenergetics. SpringerVerlag New York Inc., pp. 62-76.

Gomes, A.P., Duarte, F.V., Nunes, P., Hubbard, B.P., Teodoro, J.S., Varela, A.T., Jones, J.G., Sinclair, D.A., Palmeira, C.M., Rolo, A.P., 2012. Berberine protects against high fat diet-induced dysfunction in muscle mitochondria by inducing SIRT1-dependent mitochondrial biogenesis. Biochim. Biophys. Acta 1822 (185), 195.

Gornall, A.G., Bardawill, C.J., David, M.M., 1949. Determination of serum proteins by means of the biuret reaction. J. Biol. Chem. 177, 751-766.

Haque, M., Sanyal, A.J., 2002. The metabolic abnormalities associated with nonalcoholic fatty liver disease. Best Pract. Res. Clin. Gastroenterol. 16, 709-731.

Haslam, D.W., James, W.P.T., 2005. Obesity. Lancet 366, 1197-1209.

Hirschey, M., Shimazu, T., Goetzman, E., Jing, E., Schwer, B., Lombard, D., Grueter, C., Harris, C., Biddinger, S., Ilkayeva, O., 2010. SIRT3 regulates fatty acid oxidation via reversible enzyme deacetylation. Nature 464, 121.

Hirschey, M.D., Shimazu, T., Jing, E., Grueter, C.A., Collins, A.M., Aouizerat, B., Stančáková, A., Goetzman, E., Lam, M.M., Schwer, B., Stevens, R.D., Muehlbauer, M.J., Kakar, S., Bass, N.M., Kuusisto, J., Laakso, M., Alt, F.W., Newgard, C.B., Farese, R.V., Kahn, C.R., Verdin, E., 2011. SIRT3 deficiency and mitochondrial protein hyperacetylation accelerate the development of the metabolic syndrome. Mol. Cell 44, 177-190.

Huang, J.-Y., Hirschey, M.D., Shimazu, T., Ho, L., Verdin, E., 2010. Mitochondrial sirtuins. Biochim. Biophys. Acta 1804, 1645-1651.

Kamo, N., Muratsugu, M., Hongoh, R., Kobatake, Y., 1979. Membrane potential of mitochondria measured with an electrode sensitive to tetraphenyl phosphonium and relationship between proton electrochemical potential and phosphorylation potential in steady state. J. Membr. Biol. 49, 105-121.

Kelley, D.E., He, J., Menshikova, E.V., Ritov, V.B., 2002. Dysfunction of mitochondria in human skeletal muscle in type 2 diabetes. Diabetes 51, 2944-2950.

Kendrick, A.A., Choudhury, M., Rahman, S.M., McCurdy, C.E., Friederich, M., Van Hove, J.L.K., Watson, P.A., Birdsey, N., Bao, J., Gius, D., Sack, M.N., Jing, E., Kahn, C.R., Friedman, J.E., Jonscher, K.R., 2011. Fatty liver is associated with reduced SIRT3 activity and mitochondrial protein hyperacetylation. Biochem. J. 433, 505-514.

Kim, S.H., Shin, E.-J., Kim, E.-D., Bayaraa, T., Frost, S.C., Hyun, C.-K., 2007. Berberine activates GLUT1-mediated glucose uptake in 3T3-L1 adipocytes. Biol. Pharm. Bull. 30, 2120-2125.

Kong, W.-J., Zhang, H., Song, D.-Q., Xue, R., Zhao, W., Wei, J., Wang, Y.-M., Shan, N., Zhou, Z.-X., Yang, P., You, X.-F., Li, Z.-R., Si, S.-Y., Zhao, L.-X., Pan, H.-N., Jiang, J.-D., 2009. Berberine reduces insulin resistance through protein kinase C-dependent up-regulation of insulin receptor expression. Metab. Clin. Exp. 58, 109-119.

Kong, X., Wang, R., Xue, Y., Liu, X., Zhang, H., Chen, Y., Fang, F., Chang, Y., 2010. Sirtuin 3, a new target of PGC-1 $\alpha$, plays an important role in the suppression of ROS and mitochondrial biogenesis. PLoS One 5, e11707.

Kuda, O., Jelenik, T., Jilkova, Z., Flachs, P., Rossmeisl, M., Hensler, M., Kazdová, L., Ogston, N., Baranowski, M., Gorski, J., Janovská, P., Kus, V., Polak, J., Mohamed-Ali, V., Burcelin, R., Cinti, S., Bryhn, M., Kopecky, J., 2009. n - 3 fatty acids and rosiglitazone improve insulin sensitivity through additive stimulatory effects on muscle glycogen synthesis in mice fed a highfat diet. Diabetologia 52, 941-951.

Lee, Y., Kim, W., Kim, K., Yoon, M., Cho, H., Shen, Y., Ye, J., Lee, C., Oh, W., Kim, C., 2006. Berberine, a natural plant product, activates AMP-activated protein kinase with beneficial metabolic effects in diabetic and insulin resistant states. Diabetes 55, 2256.

Leng, S.H., Lu, F.E., Xu, L.J., 2004. Therapeutic effects of berberine in impaired glucose tolerance rats and its influence on insulin secretion. Acta Pharmacol. Sin. 25, 496-502.

Levine, R., Haft, D.E., 1970. Carbohydrate homeostasis. N. Engl. J. Med. 283, 237-246.
Liu, X., Li, G., Zhu, H., Huang, L., Liu, Y., Ma, C., Qin, C., 2010. Beneficial effect of berberine on hepatic insulin resistance in diabetic hamsters possibly involves in SREBPs, LXR $\alpha$ and PPAR $\alpha$ transcriptional programs. Endocr. J. 57, 881-893.

Lu, S.-S., Yu, Y.-L., Zhu, H.-J., Liu, X.-D., Liu, L., Liu, Y.-W., Wang, P., Xie, L., Wang, G.-J., 2009. Berberine promotes glucagon-like peptide-1 (7-36) amide secretion in streptozotocin-induced diabetic rats. J. Endocrinol. 200, 159-165.

Palmeira, C.M., Wallace, K.B., 1997. Benzoquinone inhibits the voltage-dependent induction of the mitochondrial permeability transition caused by redox-cycling naphthoquinones. Toxicol. Appl. Pharmacol. 143, 338-347.

Palmeira, C.M., Moreno, A.J., Madeira, V., 1994. Interactions of herbicides 2, 4-D and dinoseb with liver mitochondrial bioenergetics. Toxicol. Appl. Pharmacol. 127, 50-57.

Peixoto, F., Vicente, J., Madeira, V., 2004. A comparative study of plant and animal mitochondria exposed to paraquat reveals that hydrogen peroxide is not related to the observed toxicity. Toxicol. In Vitro 18, 733-739.

Pessayre, D., 2007. Role of mitochondria in non-alcoholic fatty liver disease. J. Gastroenterol. Hepatol. 22 (Suppl. 1), S20-S27.

Pessayre, D., Berson, A., Fromenty, B., 2001. Mitochondria in steatohepatitis. Semin. Liver Dis. 21, 57-69.

Rolo, A.P., Oliveira, P.J., Moreno, A.J., Palmeira, C.M., 2000. Bile acids affect liver mitochondrial bioenergetics: possible relevance for cholestasis therapy. Toxicol. Sci. 57 177-185.

Shah, P., Vella, A., Basu, A., Basu, R., Adkins, A., Schwenk, W.F., Johnson, C.M., Nair, K.S. Jensen, M.D., Rizza, R.A., 2003. Elevated free fatty acids impair glucose metabolism in women: decreased stimulation of muscle glucose uptake and suppression of splanchnic glucose production during combined hyperinsulinemia and hyperglycemia. Diabetes 52, 38-42.

Singer, T.P., 1974. Determination of the activity of succinate, NADH, choline, and alphaglycerophosphate dehydrogenases. Methods Biochem. Anal. 22, 123-175.

Tauriainen, E., Luostarinen, M., Martonen, E., Finckenberg, P., Kovalainen, M., Huotari, A., Herzig, K.-H., Lecklin, A., Mervaala, E., 2011. Distinct effects of calorie restriction and resveratrol on diet-induced obesity and fatty liver formation. J. Nutr. Metab. 2011, 525094

Teodoro, J., Rolo, A.P., Oliveira, P.J., Palmeira, C.M., 2006. Decreased ANT content in Zucker fatty rats: relevance for altered hepatic mitochondrial bioenergetics in steatosis. FEBS Lett. 580, 2153-2157.

Teodoro, J.S., Rolo, A.P., Duarte, F.V., Simões, A.M., Palmeira, C.M., 2008. Differential alterations in mitochondrial function induced by a choline-deficient diet: understanding fatty liver disease progression. Mitochondrion 8, 367-376.

The Global Burden | International Diabetes Federation, 2013. [WWW Document].idf.org. (URL http://www.idf.org/diabetesatlas/5e/regional-overviews (last accessed 5.20 2013)).

Valdecantos, M.P., Pérez-Matute, P., González-Muniesa, P., Prieto-Hontoria, P.L., MorenoAliaga, M.J., Martínez, J.A., 2012. Lipoic acid improves mitochondrial function in nonalcoholic steatosis through the stimulation of sirtuin 1 and sirtuin 3. Obesity (Silver Spring) 20, 1974-1983.

Wang, C., Li, J., Lv, X., Zhang, M., Song, Y., Chen, L., Liu, Y., 2009a. Ameliorative effect of berberine on endothelial dysfunction in diabetic rats induced by high-fat diet and streptozotocin. Eur. J. Pharmacol. 620, 131-137.

Wang, Y., Huang, Y., Lam, K.S., Li, Y., Wong, W.T., Ye, H., Lau, C.W., Vanhoutte, P.M., Xu, A., 2009b. Berberine prevents hyperglycemia-induced endothelial injury and enhances vasodilatation via adenosine monophosphate-activated protein kinase and endothelial nitric oxide synthase. Cardiovasc. Res. 82, 484-492.

Wang, Y., Campbell, T., Perry, B., 2011. Hypoglycemic and insulin-sensitizing effects of berberine in high-fat diet-and streptozotocin-induced diabetic rats. Metabolism 60, 298-305.

Xia, X., Yan, J., Shen, Y., Tang, K., Yin, J., Zhang, Y., Yang, D., Liang, H., Ye, J., Weng, J., 2011 Berberine improves glucose metabolism in diabetic rats by inhibition of hepatic gluconeogenesis. PLoS One 6, e16556.

Yin, J., Zhang, H., Ye, J., 2008. Traditional Chinese medicine in treatment of metabolic syndrome. Endocr. Metab. Immune Disord. Drug Targets 8, 99-111.

Zhang, Y., Li, X., Zou, D., Liu, W., Yang, J., Zhu, N., Huo, L., Wang, M., Hong, J., Wu, P., 2008 Treatment of type 2 diabetes and dyslipidemia with the natural plant alkaloid berberine. J. Clin. Endocrinol. Metab. 93, 2559-2565.

Zhang, H., Wei, J., Xue, R., Wu, J.-D., Zhao, W., Wang, Z.-Z., Wang, S.-K., Zhou, Z.-X., Song D.-Q., Wang, Y.-M., Pan, H.-N., Kong, W.-J., Jiang, J.-D., 2009. Berberine lowers blood glucose in type 2 diabetes mellitus patients through increasing insulin receptor expression. Metabolism 59, 285-292.

Zhou, J.Y., Zhou, S.-W., 2011. Protective effect of berberine on antioxidant enzymes and positive transcription elongation factor b expression in diabetic rat liver. Fitoterapia 82, 184-189.

Zhou, S., Palmeira, C., Wallace, K., 2001. Doxorubicin-induced persistent oxidative stress to cardiac myocytes. Toxicol. Lett. 121, 151-157.

Zhou, L., Yang, Y., Wang, X., Liu, S., Shang, W., Yuan, G., Li, F., Tang, J., Chen, M., Chen, J., 2007. Berberine stimulates glucose transport through a mechanism distinct from insulin. Metabolism 56, 405-412.

Zhou, L., Wang, X., Shao, L., Yang, Y., Shang, W., Yuan, G., Jiang, B., Li, F., Tang, J., Jing, H., Chen, M., 2008. Berberine acutely inhibits insulin secretion from beta-cells through 3',5-cyclic adenosine 5'-monophosphate signaling pathway. Endocrinology 149, 4510-4518.

Zhou, J., Zhou, S., Tang, J., Zhang, K., Guang, L., Huang, Y., Xu, Y., Ying, Y., Zhang, L., Li, D., 2009. Protective effect of berberine on beta cells in streptozotocin- and highcarbohydrate/high-fat diet-induced diabetic rats. Eur. J. Pharmacol. 606, 262-268.

Zhou, L., Wang, X., Yang, Y., Wu, L., Li, F., Zhang, R., Yuan, G., Wang, N., Chen, M., Ning, G., 2010. Berberine attenuates cAMP-induced lipolysis via reducing the inhibition of phosphodiesterase in 3T3-L1 adipocytes. BBA - Molecular Basis of Disease. 1-9. 